\title{
Design and Implementation of a Home Automation System Based on Wi-Fi
}

\author{
Rajashree Chatterjee $^{1}$, Arijit Sarkar ${ }^{2}$, Shalabh Agarwal ${ }^{3}$, Asoke Nath ${ }^{4}$ \\ Department of Computer science, St. Xavier's College, Kolkata, Kolkata, India \\ ${ }^{1}$ chattopadhyayrajashree@gmail.com, ${ }^{2}$ arijits95@gmail.com, ${ }^{3}$ shalabh@sxccal.edu, \\ ${ }^{4}$ asokejoy1@gmail.com
}

\begin{abstract}
This paper presents a design and prototype implementation of new home automation system that uses Wi-Fi technology as a network infrastructure connecting its parts. With the help of a web server and a LAN connection the system provides a scalable and a wide range coverable device. The system is highly advanced as it uses a cheaper Wi-Fi connection and a server that can store data. Users and system administrator can locally (LAN) or remotely (internet) manages and control system code. Thus, a highly automated system is available to the user. The system is secured as the user has to enter password and that is not known to any intruders.
\end{abstract}

Keywords: home automation, microcontroller, web server module, wi-fi.

\section{INTRODUCTION}

The main objective of the paper is to design an advanced home automation system based on Wi-Fi technology where the devices can be enabled or disabled using a Personal Computer (PC) or Laptop or Smart Phones through Wi-Fi. This technology finds its importance in the field of technology due to its user friendly nature. The technology serves well with the replacement of the traditional switch systems.

Wireless Fidelity (Wi-Fi) is an advanced automation system that uses radio frequency to transmit data through the air. With an initial speed of $1 \mathrm{mbps}$ to $2 \mathrm{mbps}$, it transmits data with a frequency band of $2.4 \mathrm{GHz}$ thus establishing the concept of frequency division multiplexing technology and ranges from 40-300 feet. The devices are mainly controlled through a microcontroller. The data is sent from the PC over Wi-Fi and will be received by Wi-Fi module connected to the microcontroller which reads the data and decides the switching mode of the electrical devices connected to it through Relay Boards. The microcontroller is programmed using $\mathrm{C}++$ language.

Some of the advantages of wireless technology over wired technology:

- Due to its non necessity of cabling, the installation cost is reduced to a greater extent.

- In adjacent or remote places, wireless nodes can be mounted everywhere. This helps in enlarging the covered area.

- System is scalable and the extension is easy as it does not need any physical support.

- Placement of wireless nodes is very easy as it can be installed at any location such as glass buildings or historical buildings.

- Any number of mobile devices can be attached with the system anywhere around the world.

Typical examples include an engineer who connects to the network, performs a particular management task, and disconnects after having finished the task; or control of blinds using a remote control.

For all these reasons, wireless technology is not only an attractive choice in renovation and refurbishment, but also for new installations.

This technology does not only brings comfort while handling but also makes it easy to handle centralised systems such as heating, ventilation, air condition and lighting in large MNCs. Hence they reduce cost as well as is energy saving in nature. 


\section{Algorithm}

$I^{*}$ the Main() calls all the other functions in order to start the process and to send and receive the data from the web server*/

\section{Algorithm Main()}

Step 1: Reset the ESP module.

Step 2: Set the Baud rate for both ESP module and PC.

Step 3: Configure Server by calling function start Server().

Step 4: Loop until a GET or POST request is received from a web browser.

Step 5: On receiving a GET or a POST request call the function Read Web Data().

Step 6: After the data has been read from Step 5, a call is made to the function sendPage()which sends the necessary webpage to the user.

Step 7: Goto Step 4 unless the Microcontroller LPC1768 is rebooted or switched off manually.

/* The startServer()configures the ESP as a web server */

\section{Algorithm start Server()}

Step 1: Send "AT" command to the ESP module by calling the function send CMD().

Step 2: Again send "AT" command to the ESP module by calling the function send CMD().

Step 3: Send "AT+RST" command to the ESP module to reset it by calling the function send $\mathrm{CMD}()$.If "OK"reply is received then proceed to the next step else go to Step 10.

Step 4: Sent "AT+CWJAP=<SSID $>,\langle$ PASSWORD $>$ " command to the ESP module to connect it to our WiFi router by calling the function send $\mathrm{CMD}()$.

Step 5: Sent "AT+CWMODE=3" command to the ESP module to set it both as a Station and an Access point by calling the function send $\operatorname{CMD}()$.

Step 6: Sent "AT+CWMUX=1" command to the ESP module to enable multiple connections by calling the function send $\operatorname{CMD}()$.

Step 7: Sent "AT+CIPSERVER=1, $<$ PORT $>$ " command to the ESP module to enable server mode and assign port number by calling the function send $\operatorname{CMD}()$.

Step 8: Sent "AT+CIFSR" command to the ESP module to list the Static IP of the server by calling the function send $\mathrm{CMD}()$.

Step 9: Wait Unless an IP is recieved.

Step 10: Display a message that there is a problem with the power connections.

Step 11: Exit

/* The ReadWebData() reads the GET or POST header*/

\section{Algorithm ReadWebData()}

Step 1: Read the +IPD section of the GET or POST header.

Step 2: Set logout variable depending upon whether Logout button was clicked or not.

Step 3: Set login variable depending upon whether Login button was clicked or not.

Step 4: If the request is a GET request then send Login Page and go to Step 9, otherwise go to next step.

Step 5: If login flag is set then go to next step 6 else go to Step 7.

Step 6: If <username> and <password> are correct then set send Activity Page flag and reset wrong flag, otherwise set send Login Page and wrong flags.

Step 7: If logout flag is set then set send Login Page flag and go to Step 9. 
Step 8: Read the POST data and set send Activity Page.

Step 9: Exit

/*The sendPage()preparesthe ESP for sending dataand decides which webpage to send depending on user's need*/

\section{Algotithm sendPage()}

Step 1: If sendLoginPage flag is set, then copy the Login page HTML code into webpage buffer.

Step 2: If sendActivityPage flag is set, then copy the Activity page HTML code into webpage buffer.

Step 3: Calculate Buffer length of webpage and send "AT+CIPSEND $=<$ Channel $>,<$ Buffer Length $>$ " to the ESP.

Step 4: Send Webpage by making a call to the function sendWEB().

Step 5: If webpage is send successfully then call sendCheck() function.

Step 6: Exit

/*The sendWEB()is used to send the webpage to the user*/

\section{Algorithm sendWEB()}

Step 1: Check whether bytes can be written on serial port connecting the LPC1768 and the ESP module.

Step 2: If the above condition holds, then write to the serial port until the end of webpage buffer is reached.

Step 3: Exit

/*The sendCheck()closes the channel used to send webpage data to the user*/

\section{Algotithm sendCheck()}

Step 1: Send "AT+CIPCLOSE=<Channel $>$ " to the ESP module to close the channel via which it sends the webpage to the user.

Step 2: Exit.

l*The sendD() establishes a serial connection between the microcontroller and the server module*/

\section{Algorithm sendCMD ()}

Step 1: Write the cmdBuf buffer holding the AT commands onto the serial port connecting the LPC1768 and ESP module.

Step 2: Exit.

/*The getReply() fetches the response from the server to the microcontroller*/

\section{Algorithm getReply()}

Step 1: Empty the replyBuf.

Step 2: Reset and start the timer.

Step 3: Loop until timer value is within the timeout value

Step 4: If serial port is readable, then read each byte into the replyBuf.

Step 5: Exit.

/*The getTemp() calculates the temperature*/

\section{Algorithm getTemp ()}

Step 1: Loop ten times to take ten readings from LM35 sensor and place it inside array a.

Step 2: Take the average of all the elements of the array a.

Step 3: Calculate the temperature in Celsius.

Step 4: Exit. 


\section{TECHNICAL DESCRIPTION}

We have used different hardware components mainly to design the circuit. The following are the list of components:

1. The microcontroller LPC1768 which is an ARM processor and is also known as CORTEX M3 processor. It is a 32-bit processor for low power, cost-sensitive, highly deterministic real-time embedded applications. The processor is highly configurable enabling a wide range of implementations from those requiring memory protection and powerful trace technology to cost sensitive devices requiring minimal area.

Key advantages:

- It is designed such that the chips are placed well with a high performance and power with new system interface.

- Captures a large market over the world due to its easy to develop system features.

- It is having the features such as integrated software controlled sleep modes, extensive clock gating and optional state retention

2. The web server module ESP8266 when integrated with the microcontroller can access Wi-Fi under TCP/IP module.

Key advantages:

- P2P Wi-Fi Direct

- $1 \mathrm{MB}$ Flash Memory

- TCP/IP Protocol stack(integrated)

- Less than $10 \mu \mathrm{A}$ power down leakage

- 32 bit CPU can also be used as Application Processor

- UART approved

- $802.11 \mathrm{~b} / \mathrm{g} / \mathrm{n}$

- Capacity of Stand By Power Consumption

3. The device driver module CP2102 is a highly integrated USB-to-UART Bridge controller that connects the microcontroller to the Wi-Fi module.

4. 2 channel Relay switches are used to control the circuit using low power signal. It is a 5V DC that acts as an amplifier that repeats the signals coming from one circuit and transmits it to the devices.

5. The LM35 is an integrated-circuit temperature measuring device that senses the temperature of $40{ }^{\circ} \mathrm{C}$ to $110^{\circ} \mathrm{C}$ and operates with $4 \mathrm{~V}$ minimum voltage.

There are also some of the software requirements as mentioned below:

1. mbed online compiler: It is a platform where we can develop a $\mathrm{C}++$ based program by which we can control the microcontroller. It is totally free of cost and we can write and modify the programs as per requirement. It also can handle errors and has an online compiler that compiles the program.

2. Flash Magic: It is software that is developed by the Embedded systems that helps to burn the program inside the microcontroller. Erasing of data, reading and programming the flash memory, adjust the Boot Vector and many other features can be handled using this software.

3. CP2102 Driver: This driver sets up a serial communication between the microcontroller and the Wi-Fi module.

4. BintoHex Converter: It is a software the simply converts the binary code into HEX code which is used to burn inside the microcontroller. 


\section{SYSTEM DESIGN AND IMPLEMENTATION}

A. Proposed Home Automation System layout

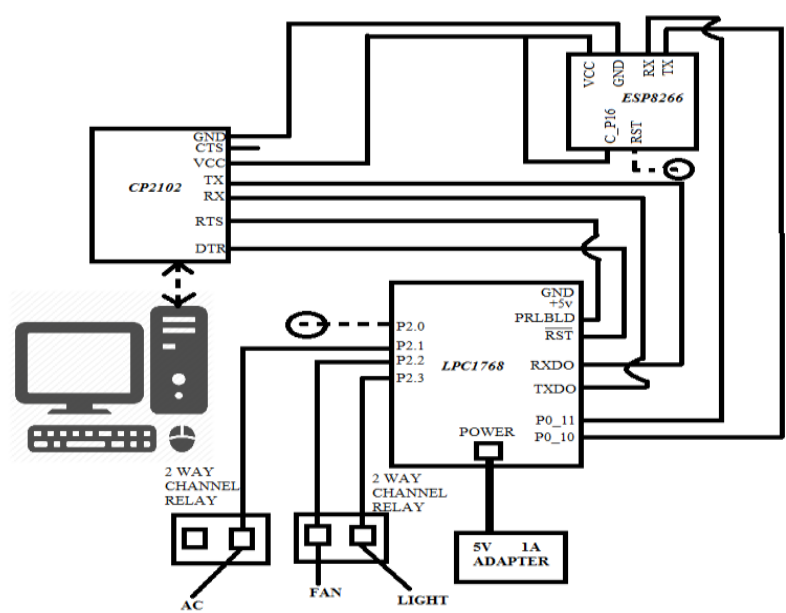

Figure1. Circuit diagram of Home Automation

B. Working

- A FLY PORT and a FLY PORT NEST to implement the on/off action using the microcontroller LPC1768 and the Microchip ESP8266- Wi-Fi Module.

- A common Router is used to connect the devices between them using Wi-Fi.

- Ad hoc connectivity allows Point to Point connection through wireless nodes without any Access Points.

- Web server from the cloud can be hosted from any devices to enable the system.

C. Assumptions and Dependencies

- Only one administrator at a time can handle the system.

- Proper and popular browsers are to be used.

- Proper hardware components are to be used.

- Any OS can be used to run the server.

- Proper internet connection must be used.

\section{RESUlts AND Discussion}

\section{A. Results:}

Case 1

Input: When wrong Username or password is entered by the user.

Output: A message showing the error is displayed.

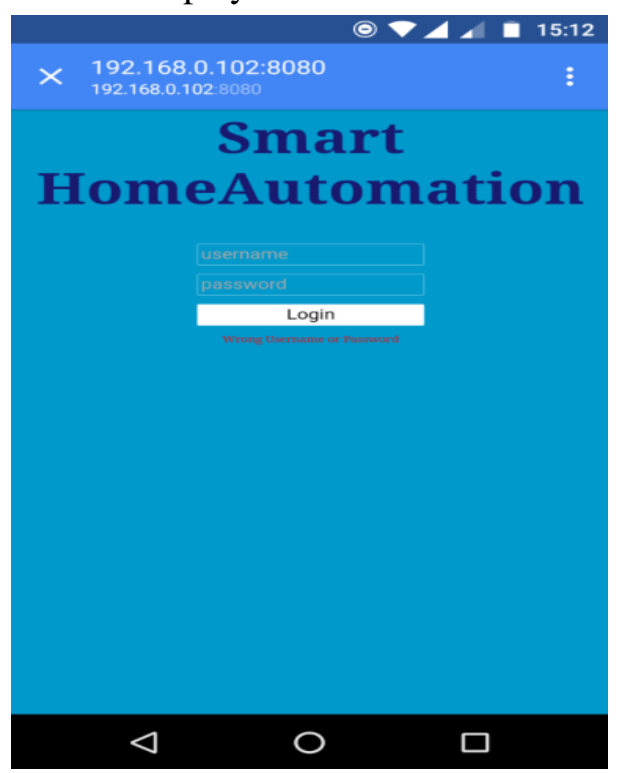

Figure2. Message showing wrong input 
Rajashree Chatterjee et al.

Case 2

Input: When Light and AC is enabled on the webpage.

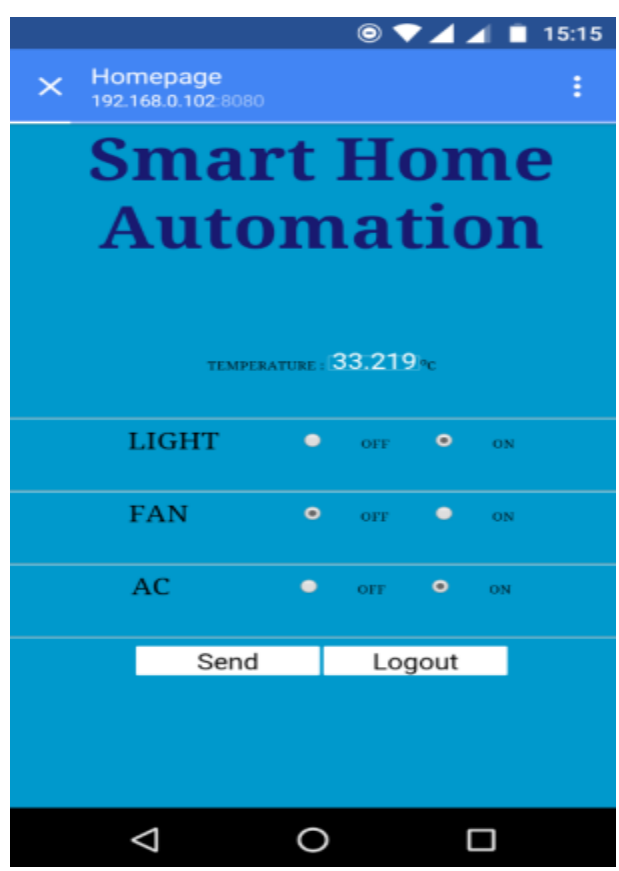

Figure3. Light and AC is selsected

Output: Light and AC are switched on.

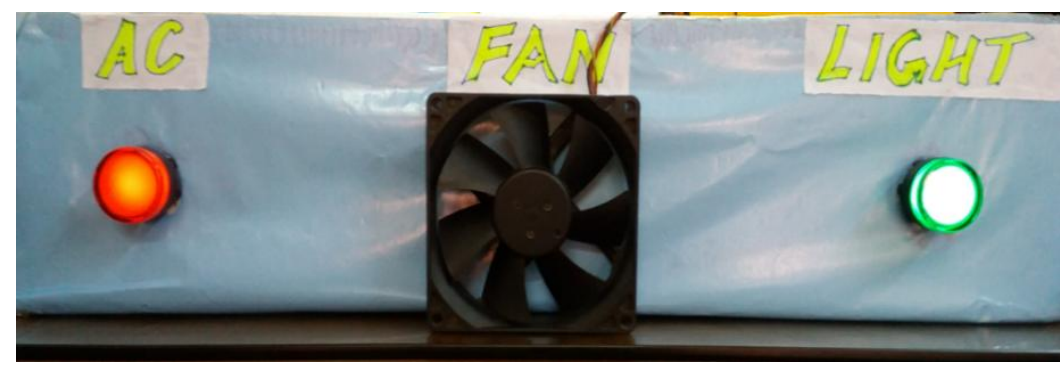

Figure4. Light and Ac is enabled

Case 3

Input: When Light, Fan and AC are all enabled.

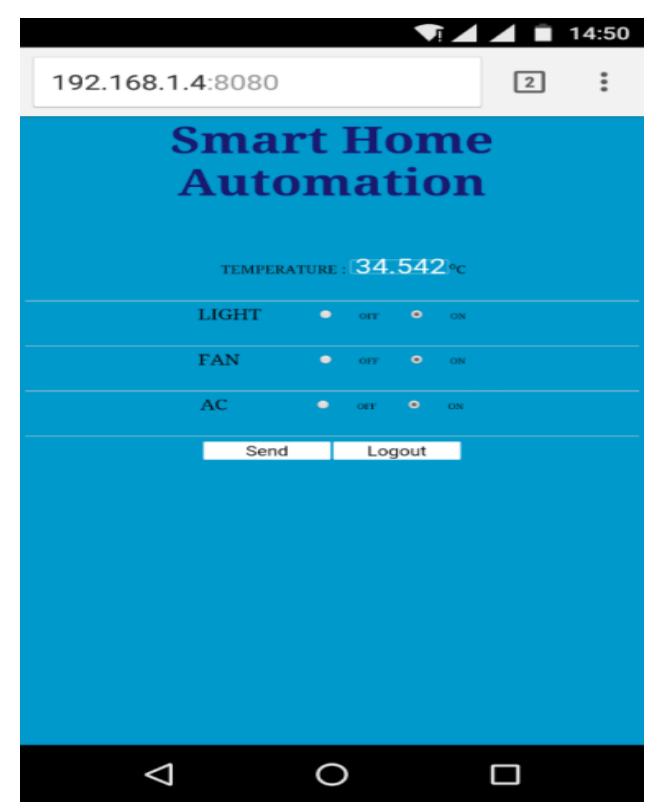

Figure5. Light, Fan and AC are all switched on 
Output: All the three devices are switched on.

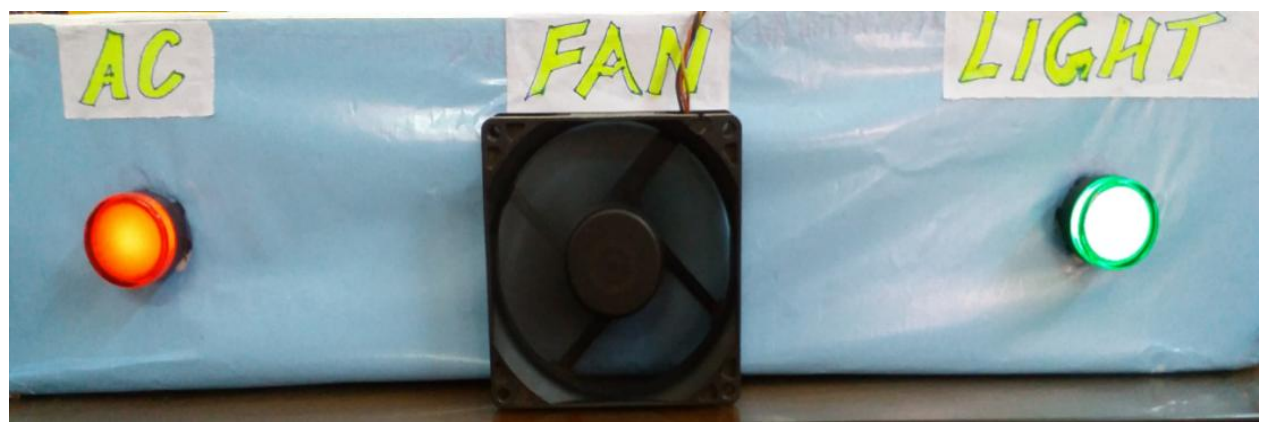

Figure6. Light, Fan and $A C$ are all enabled

Case 4

Input: Logged into the system from an external network and disabled all devices.

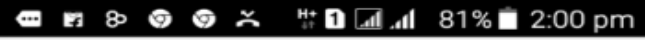

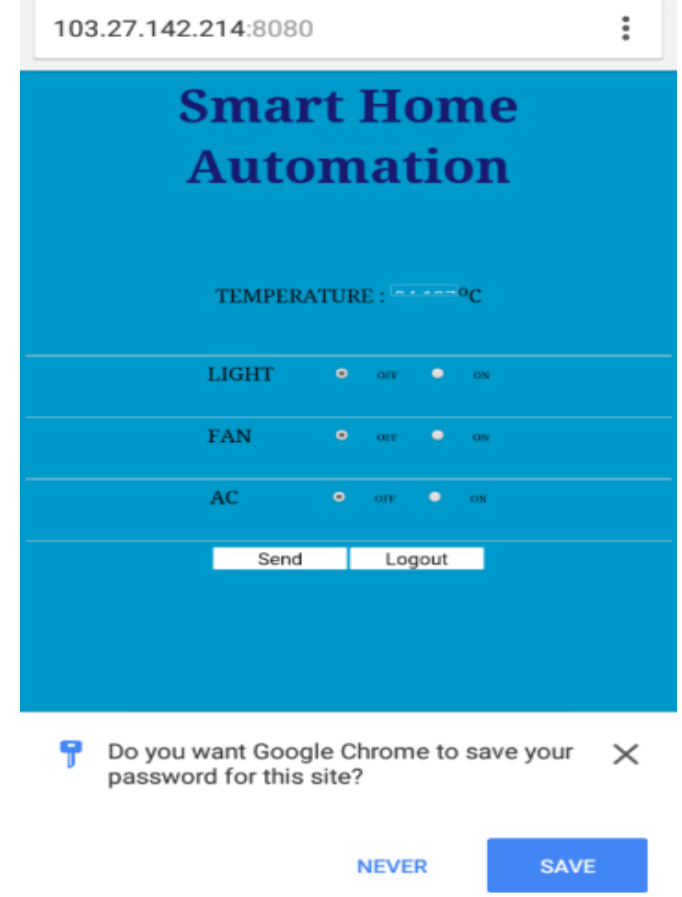

Figure7. All devices are switched off

Output: The three devices are switched off.

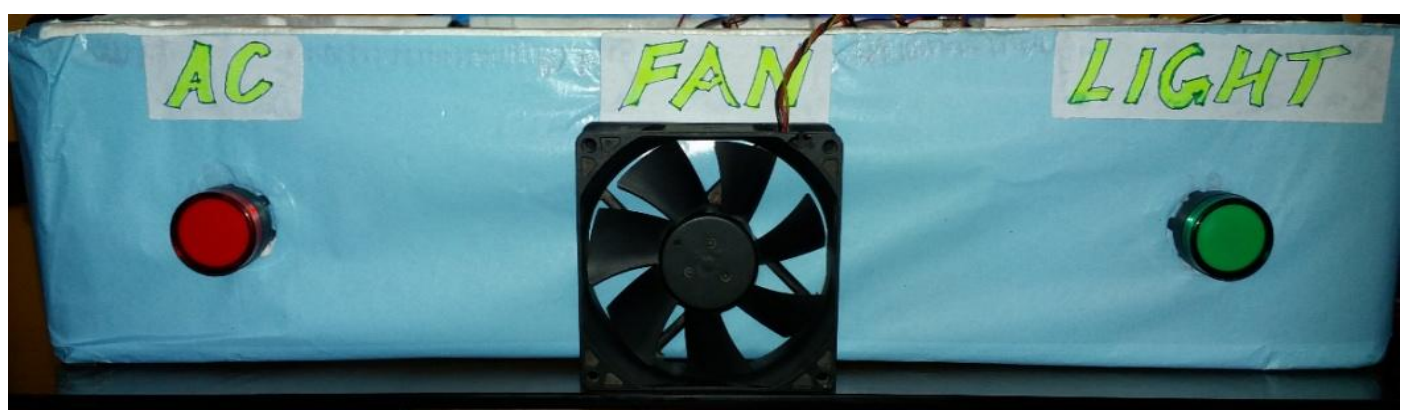

Figure8. All devices are disabled

\section{B. Discussion:}

We have done port forwarding in the router so that anyone who is outside the Wi-Fi network can access the system using other networks. We have used the port 8080 which has the user login page and the activity page. The user when connects from other network will have to use the port number with the IP address and can access the web pages and the devices from any corner of the world. 
The following figure 9 shows that the page has been accessed from different network by using the port number.

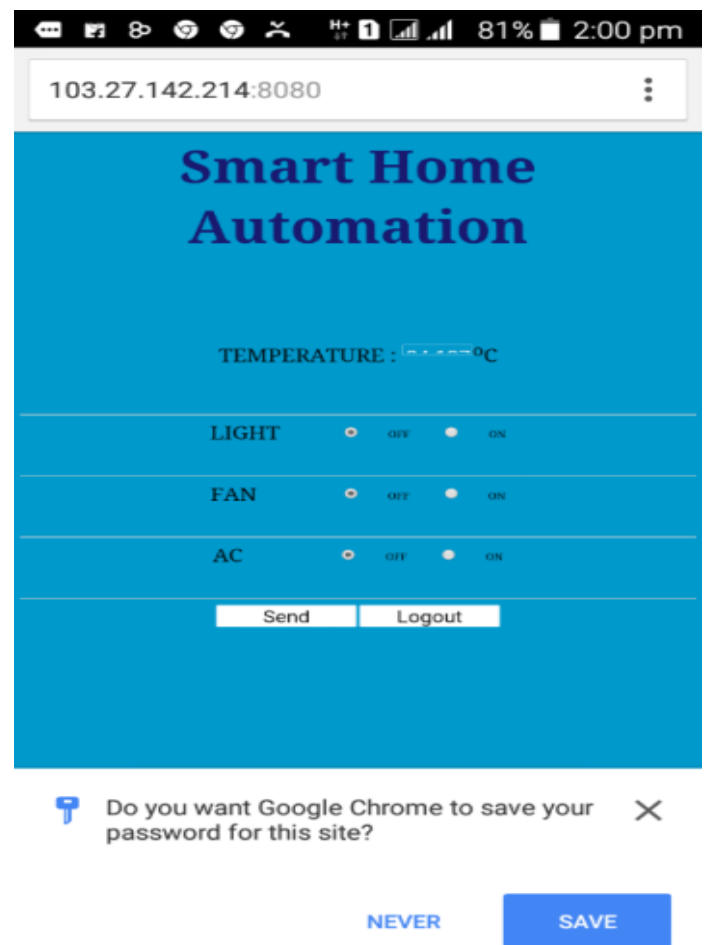

Figure9. Shows the availability of the page using port forwarding from any network and any place

There are two limitations in the present system and they are:

- Firstly, the data sending from the server as per request was not in order. The web page ordering was totally reversed so that the data was not synchronized.

- POST request could not be read entirely, so the activity could not be determined properly.

However this drawback was later resolved by few changes in the code and the ordering was done correctly.

\section{Conclusion And Future Scope}

The proposed paper includes the implementation of a low cost, secure and remotely accessible home automation system. This uses Wi-Fi technology which has a greater acceptance in the world's market as it satisfies user needs and requirements. Wi-Fi technology has minimized the wiring to a large extent. Hence we can conclude that the required goals and objectives of home automation system have been achieved.

The following point presents the suggested future work for implemented prototype:

- More hardware modules can be integrated to the system which can communicate with sensors and actuators that use wireless technologies.

- Security cameras can be installed which will monitor the premises, allowing the user to observe activity around the house.

- It could be useful for old aged/especially able people as appliances can be controlled by merely a touch. It is also possible to control the system using voice commands using Android. It is safe because there is no chance of getting electric shock.

- Artificial Intelligence can be added to make the system more advanced.

\section{ACKNOWLEDGMENT}

This paper is based on M.Sc Post Graduation project accomplished at St. Xavier's College (Autonomous), Kolkata under University of Calcutta, Department of Computer Science, 2016. The project was supervised by the $3^{\text {rd }}$ author, the $4^{\text {th }}$ author helped in preparing this paper and the team work is done by the $1^{\text {st }}$ and the $2^{\text {nd }}$ author. 


\section{REFERENCES}

[1] Explore Embedded

[2] https://www.mbed.com/en/

[3] http://www.nxp.com/documents/data_sheet/LPC1768_66_65_64.pdf

[4] http://www.robodukkan.com/class/INNOVAEditor/assets/2_CHANNEL_5V_10A_RELAY_MO DULE.pdf

[5] https://www.silabs.com/Support\%20Documents/TechnicalDocs/an197.pdf

[6] https://cdn-shop.adafruit.com/product-files/2471/0A-ESP8266_Datasheet_EN_v4.3.pdf

[7] https://developer.mbed.org/users/4180_1/notebook/using-the-esp8266-with-the-mbed-lpc1768/

[8] Matthias Gauger,Daniel Minder,Arno Wacker, Andreas Lachenmann,"Prototyping SensorActuator Networks for Home Automation", REALWSN'08, April 1, 2008, Glasgow, United Kingdom.

\section{AUTHORS' BIOGRAPHY}

Rajashree Chatterjee, received the M.Sc degree in Computer Science from St. Xavier's College (Autonomous), Kolkata under University of Calcutta in 2016. She has done her graduation in Computer Science from Seth Anandram Jaipuria College, Kolkata under University of Calcutta in the year 2014.

Arijit Sarkar, received the M.Sc degree in Computer Science from St. Xavier's College (Autonomous), Kolkata under University of Calcutta in 2016. He has done his graduation in Computer Science from Scottish Church College, Kolkata under University of Calcutta in the year 2014.

Prof. Shalabh Agarwal is Associate Professor and Head of the Dept. of Comp.Sc., St.Xavier's College (Autonomous), Kolkata and also Director of Computer Centre with more than 23 years experience in teaching. He has the added responsibility of Systems Incharge of the College and has immense contribution in implementing innovative IT applications in the institution. Keeping up with the latest trends, he is very keen on research and has many publications in International and National journals, including a few in IEEE sponsored conferences and journals.

Dr. Asoke Nath, is Associate Professor in department of Computer Science, St. Xavier's College (Autonomous), Kolkata. Apart from his teaching assignment he is involved with various researches in cryptography and network security, Visual Cryptography, Steganography, Mathematical modelling of Social Networks, Green Computing, Big data analytics, MOOCs, Quantum computing, e-learning. He has already published more than 197 publications in reputed Journals and conference proceedings. Dr. Nath is the life member of MIR Labs (USA), CSI Kolkata Chapter. 